https://helda.helsinki.fi

\title{
The gap between behavioral risk status and willingness to change behavior among healthcare professionals
}

Kasila, K.

2018-11

Kasila , K, Hallman , M , Kautiainen , H , Vanhala , M \& Kettunen , T 2018 , ' The gap between behavioral risk status and willingness to change behavior among healthcare professionals ' , Perspectives in Public Health , vol. 138 , no. 6 , pp. 311-315 . https://doi.org/10.1177/175791391775

http://hdl.handle.net/10138/307707

https://doi.org/10.1177/1757913917751564

publishedVersion

Downloaded from Helda, University of Helsinki institutional repository.

This is an electronic reprint of the original article.

This reprint may differ from the original in pagination and typographic detail.

Please cite the original version. 


\section{The gap between behavioral risk status and willingness to change behavior among healthcare professionals}

Authors

\section{K Kasila}

Faculty of Sport and Health

Sciences, University of Jyvaskyla, PO Box 35, Fl-40014 University of Jyväskylä, Finland

Email: kirsti.kasila@jyu.fi

\section{Hallman}

Unit of Primary Health Care, Central Finland Health Care District, Jyväskylä, Finland

\section{$\mathrm{H}$ Kautiainen}

Unit of Primary Health Care, Helsinki University Central Hospital, Helsinki, Finland; Department of General Practice and Primary Health Care, University of Helsinki, Helsinki, Finland; Unit of Primary Health Care, Turku University Hospital, Turku, Finland

\section{Vanhala}

Unit of Primary Health Care, Central Finland Health Care District, Jyväskylä, Finland; Unit of Primary Health Care, University of Eastern Finland, Kuopio, Finland

\section{T Kettunen}

Faculty of Sport and Health Sciences, University of Jyvaskyla, Jyväskylä, Finland; Unit of Primary Health Care, Central Finland Health Care District, Jyväskylä, Finland

Corresponding author: Kirsti Kasila, as above

\section{Keywords}

healthcare professionals; behavioral health risk factor; health habits; ongoing change attempts

Abstract

Aims: This study explored behavioral health risk factors among healthcare professionals and investigated the at-risk persons' satisfaction with their health habits and ongoing change attempts.

Methods: The study was based on a cross-sectional web-based survey directed at the nurses and physicians $(N=1233)$ in Finnish healthcare. Obesity, low physical activity, smoking, and risky alcohol drinking were used as behavioral health risk factors.

Results: In all, 70\% of the participants had at least one behavioral risk factor, and a significant number of at-risk persons were satisfied with their health habits and had no ongoing change process. Good self-rated health and good self-rated work ability were significantly associated with whether a participant had a behavioral health risk factor.

Conclusion: Overall, unhealthy behaviors and a lack of ongoing change attempts were commonly observed among healthcare professionals. Work in healthcare is demanding, and healthy lifestyles can support coping. Thus, healthy lifestyle programs should also be targeted to healthcare professionals.

\section{INTRODUCTION}

Noncommunicable diseases (NCDs), such as cardiovascular diseases and diabetes, are major challenges worldwide. The diseases cause premature deaths, inability to work, and sickness absences. It is known that a large percentage of those diseases and disorders could be prevented by reducing behavioral risk factors, such as tobacco use, harmful use of alcohol, physical inactivity, and unhealthy diet.1,2 This study focuses on examining the risky health behaviors of healthcare professionals. There is evidence that although their habits are healthier than the general population, in some respects, they are comparable with the general population in, for example, preventive screening, physical activity, and weight control..$^{3-7}$

Healthcare professionals' tasks and responsibilities are particularly demanding, thus maintaining and promoting their wellbeing and work ability requires special attention and efforts. In previous studies, a healthy lifestyle among healthcare professionals has been associated with a good self-rated health and physical and mental wellbeing, and thereby with various positive work-related indicators such as good work ability, low sickness absence, and low retirement intentions..$^{8-10}$ Another aspect of the issue is that healthcare professionals may play an important role in intervening in the lifestyle of patients. Previous studies have shown that doctors' own health habits are strongly related to their professional health promotion practices: those who have healthy habits are more likely to provide health counseling to their patients and can be powerful role models of a healthy lifestyle. ${ }^{11-14}$ In the case of nurses, however, there is little evidence to support this effect. ${ }^{15}$

Over the last decade, more attention has been paid to the issue of healthy lifestyle behavior 
among healthcare professionals and students. 4,6,7,11,16,17 In addition, the international movement of the Health Promoting Hospitals and Services (HPH) network emphasizes the importance of addressing the health and health behavior of healthcare professionals. ${ }^{18}$ Several studies have examined healthcare professionals' behaviorrelated health risk status and their perceived barriers to a healthy lifestyle, such as feeling tired, not having time, work schedules, and family commitments. ${ }^{19,20}$ Changing one's health behavior is a multifaceted process, one that is influenced by informational, environmental, self-regulatory, and other factors. ${ }^{21}$ And just as these factors affect the general population, healthcare professionals are also influenced by them. Surrounded by work and life challenges, a mere informational approach is not enough, and it is precisely this approach that has traditionally been strong among healthcare professionals. Adopting and maintaining behavior change demands motivation, self-efficacy, social support, and self-regulatory skills, including goalsetting, action planning, self-monitoring, and self-reinforcement. ${ }^{22}$ The planning of effective lifestyle intervention begins with the identification of the target group's health behavior profile and the need for changes. This research focuses on this issue.

\section{THE AIM OF THE STUDY}

The purpose of this study was to examine behavioral health risk factors among healthcare professionals, to explore the at-risk persons' satisfaction with their health habits and ongoing change attempts.

\section{METHODS}

All employees based on a hospital campus with the Central Finland Health Care District (CFHCD), a member of the HPH Network since 1998, were invited to take part in a web-based questionnaire survey $(N=3586)$ in spring 2010 . The response rate was $54 \%$. The physicians and nurses who answered the questionnaire formed the sample of this study ( $n=1233)$. The ethical clearance for the study was obtained from the ethical committee of the respective institution.

The participants assessed their general health and work ability with a single item per issue (scoring $1=$ poor to $5=$ good). Obesity (body mass index $(\mathrm{BMl}) \geq 30$ ) was used as a behavior-related risk factor and as an indicator of the increased behavioral risk in eating habits. BMI was calculated from the participants' self-reported weight and height. Low physical activity, smoking, and risky alcohol drinking were used as behavioral health risk factors. The physical activity level was evaluated using the FIT (Frequency, Intensity, Time) Index, which is a crude measure of physical activity level based on subjective ratings of the frequency, intensity, and time (duration) of aerobic activity. ${ }^{23}$ The participants were requested to describe their physical activity with three items: (1) options for frequency of exercise (scoring 1-5 points): less than one time per month, a few times per month, 1-2 times per week, 3-5 times per week, 6 or more times per week; (2) options for intensity of exercise (scoring 1-5 points): light aerobic exercise, low to moderate aerobic and sports activities, moderate aerobic activities, moderately high aerobic activities and intermittent sports activities that result in sustained heavy breathing and perspiration, and highintensity activities that result in sustained heavy breathing and perspiration; and (3) options for time spent working out (scoring 1-4 points): less than $10 \mathrm{~min}$, 10-19 min, 20-30 min, and over $30 \mathrm{~min}$. The FIT index (scores range from 1 to 100) was calculated so that a higher value indicated a more sufficient level of physical activity. The limit for insufficient physical activity was below 37 points.

Smoking was measured with one item in the questionnaire (Do you use tobacco products - cigarettes, cigars, pipe, or snuff - yes or no?) The alcohol consumption was examined using an Alcohol Use Disorders Identification Test Consumption (AUDIT-C) Screening Questionnaire, which included three questions (How often do you have a drink containing alcohol? How many drinks containing alcohol do you have on a typical day when you are drinking? How often do you have six or more drinks on one occasion?) Each AUDIT-C question was scored with 0-4 points, resulting in a total score ranging from 0 to 12 points. The risk index was calculated so that a higher value indicated a more risky drinking behavior. In this study, the limit for risky drinking was female $\geq 5$ points and male $\geq 6$ points, indicating a need for a complete identification of drinking habits using the whole Alcohol Use Disorders Identification Test (AUDIT). 24,25

Furthermore, the participants were asked to report their satisfaction with their health habits relating to physical activity, smoking, alcohol drinking, and eating, and their ongoing change attempts relating to each habit. There were three options for responses: satisfied with a health habit, dissatisfied with a health habit but no ongoing change attempt, and dissatisfied with a health habit and ongoing change attempt.

\section{Statistical analysis}

The comparison between groups was made using a chi-square test. The differences were estimated by a generalized linear model with the binomial family, identity link, and robust standard error. Statistical analyses were conducted using the Stata v.11.0 statistical software (StataCorp, College Station, TX, USA).

\section{RESULTS}

Of the 1233 participants with complete data, $89 \%(n=1102)$ were nursing personnel and $11 \%(n=131)$ were physicians. The majority of the participants $(87 \%, n=1077)$ were female. Table 1 presents the detailed characteristics of the study population. In all, $70 \%(n=864)$ of the participants had at least one behavioral health risk factor. In these data, a greater proportion of female respondents had no behavioral risk factors when compared with male respondents. Male respondents more commonly had at least two behavioral risk factors. A large proportion of physicians had no behavioral risk factors when compared with nursing staff. Nursing staff more commonly had at least two behavioral risk factors. Good self-rated health and good self-rated work ability were significantly associated 
Table 1

Characteristics of the study population $(N=1233)$

\begin{tabular}{|c|c|c|c|c|}
\hline & \multicolumn{3}{|c|}{ Number of behavioral health risks } & \multirow[t]{3}{*}{$p$ value } \\
\hline & 0 & 1 & $\geq 2$ & \\
\hline & $n=369(\%)$ & $n=481(\%)$ & $n=383(\%)$ & \\
\hline Sex & & & & $<.001$ \\
\hline Female $(n=1077)$ & $343(32)$ & 425 (39) & $309(29)$ & \\
\hline Male $(n=156)$ & $26(17)$ & $56(36)$ & $74(47)$ & \\
\hline Age group & & & & .22 \\
\hline$<31(n=262)$ & $92(35)$ & $92(35)$ & $78(30)$ & \\
\hline $31-40(n=272)$ & $73(27)$ & $112(41)$ & 87 (32) & \\
\hline $41-50(n=359)$ & $116(32)$ & $136(38)$ & $107(30)$ & \\
\hline $51-60(n=304)$ & $81(27)$ & $122(40)$ & $101(33)$ & \\
\hline$>60(n=36)$ & $7(19)$ & $19(53)$ & $10(28)$ & \\
\hline Occupation & & & & .035 \\
\hline Physicians $(n=131)$ & $47(36)$ & $56(43)$ & $28(21)$ & \\
\hline Nursing staff $(n=1102)$ & $322(29)$ & 425 (39) & $355(32)$ & \\
\hline Self-rated health: good & $330(89)$ & $387(80)$ & $254(66)$ & $<.001$ \\
\hline $\begin{array}{l}\text { Self-rated work ability: } \\
\text { good }\end{array}$ & 327 (89) & $398(83)$ & $276(72)$ & $<.001$ \\
\hline
\end{tabular}

with whether a participant had a behavioral health risk (Table 1).

A total of $46 \%(n=569)$ of the participants were categorized as physically inactive, and $37 \%(n=211)$ of the physically inactive participants were satisfied with their level of physical activity. Among those who were dissatisfied with their level of physical inactivity, 42\% $(n=149)$ were in the process of changing their exercise habits. In all, $20 \%$ of the respondents $(n=246)$ smoked, and $51 \%(n=125)$ of the smokers were satisfied with their smoking habits. Among those respondents who smoked and were dissatisfied with their smoking habits, 31\% $(n=37)$ were in the process of giving up smoking. Alcohol consumption categorized as risky drinking was reported by $17 \%(n=209)$, and $74 \%(n=154)$ of the risky drinkers were satisfied with their drinking habits. A total of $60 \%(n=33)$ of the participants who were dissatisfied with the risky drinking situation were changing their drinking habits. Overall, $14 \%$ of respondents $(n=175)$ were categorized as obese, and $45 \%$ of the obese participants $(n=78)$ were satisfied with their eating habits. Among those who were obese and dissatisfied with their eating habits, 58\% $(n=56)$ were in the process of changing their eating habits (Figure 1). In those risky health behavior groups where the participants were dissatisfied with their health habits, ongoing change attempts were the rarest among smokers $(p<.001)$.

\section{DISCUSSION}

The findings reveal that nearly half of the healthcare professionals were not physically active enough for the benefit of their health. Levels of physical activity were better than the general Finnish population, but there was still a high percentage of inactive healthcare professionals. ${ }^{26}$ Over one-tenth were obese, alcohol consumption categorized as risky drinking was reported by $17 \%$, and one-fifth of the participants smoked. Rates of obesity and risky alcohol drinking were lower than the general population, but the smoking rate was comparable with the general Finnish population. ${ }^{26}$ Overall, more than onequarter of the participants had no risk factors, while nearly one-third of the participants had at least two behavioral health risk factors. In this regard, our findings support the notion that behavioral health risks can be accumulated together. ${ }^{3}$ 
Figure 1

Participants with a behavioral health risk, satisfaction with their health habits and ongoing change attempts

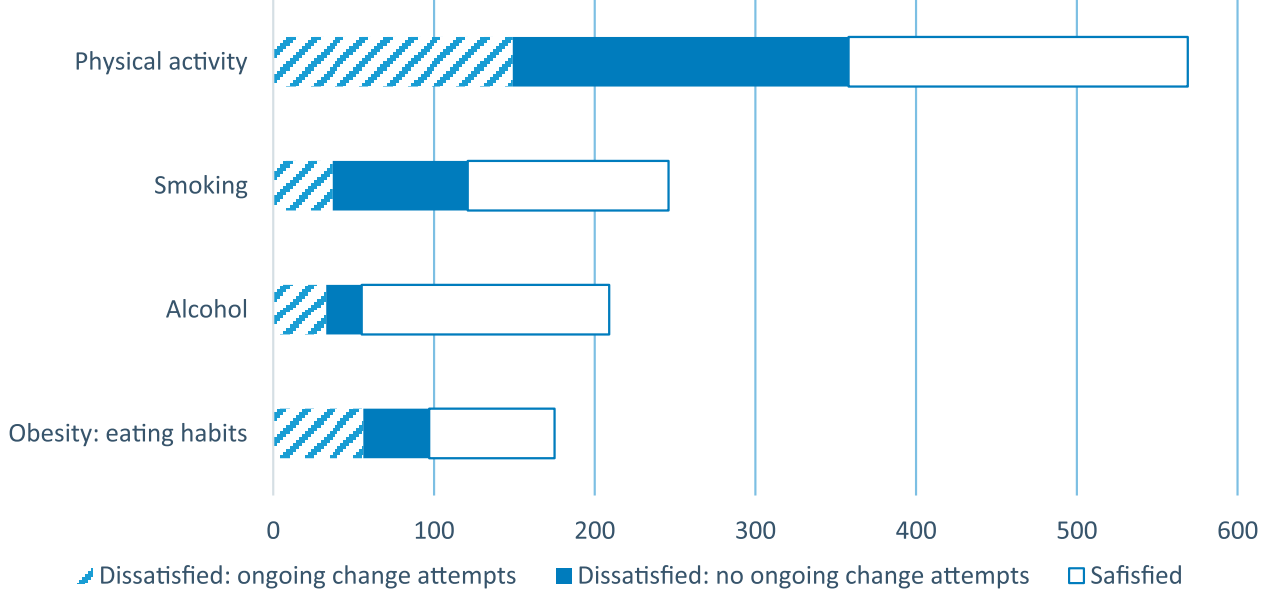

The results demonstrate that those who had behavioral health risk factors were less likely to report good general health and good work ability than those without behavioral health risks. These results are in line with those of Tountas et al., who found that healthcare professionals' protective health behaviors are associated with good general health perception and wellbeing. This association is significant because previous studies have revealed that good self-rated health, among other things, decreases the risk of future long-term sickness and the likelihood of retirement intentions among healthcare professionals. 8,9

The results also reveal that between one-third and three-quarters of those with a particular behavioral health risk were satisfied with their health habits and were not considering or implementing a change process. Only a minority of those with a health risk appeared to be in the process of changing their health habits. The at-risk persons' satisfaction with their health habits is an interesting finding and it calls for further discussion. In this study, the participants were nursing and physician professionals, who should know the negative consequences of behavioral health risk factors. They also ought to know that a large percentage of NCDs could be prevented by reducing these risk factors. ${ }^{1,2}$ It is assumed that knowledge plays an important role in the perception of behavioral risk factors and in the needs assessment, which naturally precedes concrete behavior changes. ${ }^{22}$ The results of this study showed, in line with $\mathrm{Mo}$ et al., that mere professional knowledge is not enough to produce personal behavior changes among healthcare professionals. Instead of knowledge, reinforced self-efficacy and efforts to reduce barriers have also been found to be crucial variables in improving health behavior among healthcare professionals. ${ }^{19,20}$

The phenomenon is, in many ways, a complex one. Having professional knowledge and awareness of one's own health risk status produces contradictory feelings for the at-risk healthcare professionals. ${ }^{27}$ It may be easier to combat the necessity of behavioral change and try to maintain a sense of satisfaction than to attempt to change unhealthy habits. Conflicting thoughts can also arise from situations in which the healthcare professionals counsel patients to change their behavioral health risk factors, and at the same time, they are satisfied with their own unhealthy behavior. This unpleasant situation may be reflected in healthcare professionals' health counseling practices. It is known that especially doctors who have healthy habits are more likely to counsel patients on healthy habits. ${ }^{11-13}$

The study has some limitations. The survey data were collected from a single hospital district only, meaning the results may not be generalizable to a larger population of healthcare professionals. However, the number of respondents was moderate and the demographic profile of the data was comparable with that of the CFHCD. The proportion of men among the respondents was small, but it was comparable with the number of male workers in Finnish healthcare. ${ }^{28}$ The average age of healthcare workers in Finland is about 43 years. ${ }^{28}$ In this study, half of the respondents' ages were between 30 and 50 years.

The respondents may differ from the non-respondents, but the hospital personnel information system did not allow the non-respondents' background information to be analyzed. It could be assumed that the voluntary respondents have healthy habits or that they have the willingness to change unhealthy habits. However, this study reached a large number of at-risk persons who did not have willingness to change their unhealthy behavior. Most of the study respondents belonged to nursing staff, and it was not seen as necessary to compare each health habit between different occupational groups, because the health promotion intervention was intended to address defined risk limits rather than to target specific occupations. Self-reported information may also have presented recall biases and assessing satisfaction with health 
habits includes subjective interpretations. In this study, the different risk groups were determined according to generally defined risk limits. However, it is difficult to determine a risky limit for eating habits. The decision to use obesity as an indicator of eating habit behavioral risk is problematic because obesity can be associated with physical activity and other factors. ${ }^{29}$

\section{CONCLUSION}

The results revealed that a significant number of participants had behavioral health risk factors. In each behavioral risk group, a relatively large proportion of participants were satisfied with their health habits and were not considering a change process or currently implementing one. These findings indicate the importance of increasing efforts to improve healthcare professionals' healthy lifestyle behavior, which would have benefits for both work and wellbeing. The current findings show that workplace health promotion activities should primarily focus on physical activity and smoking cessation. Participation in structured, well-planned workplace health promotion activities in which mediating psychosocial factors and experienced barriers are targeted may improve the health behavior of employees in healthcare settings. ${ }^{19,20,30,31}$

\section{CONFLICT OF INTEREST}

The author(s) declared no potential conflicts of interest with respect to the research, authorship, and/or publication of this article.

\section{ETHICAL CONSIDERATIONS}

Ethical clearance for the study was obtained from the Ethical Committee of University of Jyväskylä. Informed consent from participants was obtained with the online survey in the first survey field as a condition for continuing.

\section{FUNDING}

The author(s) received no financial support for the research, authorship, and/or publication of this article.

\section{References}

1. World Health Organization. Global status report on noncommunicable diseases 2010 Geneva: World Health Organization; 2011.

2. World Health Organization. Global action plan for the prevention and control of noncommunicable diseases 2013-2020. Geneva: World Health Organization; 2013.

3. Blake H, Mo PK, Lee S et al. Health in the NHS: lifestyle behaviours of hospital employees. Perspect Public Health 2012;132(5):213-5.

4. Chevan J, Haskvitz EM. Do as I do: exercise habits of physical therapists, physical therapist assistants, and student physical therapist. Phys Ther 2010;90(5):726-34.

5. Frank E, Segura C. Health practices of Canadian physicians. Can Fam Physician 2009;55(8):810-11.e7.

6. Kenna GA, Wood MD. Alcohol use by healthcare professionals. Drug Alcohol Depend 2004;75(1):107-16.

7. Bazargan M, Makar M, Bazargan-Hejazi S et al. Preventive, lifestyle, and personal health behaviors among physicians. Acad Psychiatry 2009;33(4):289-95.

8. Heponiemi T, Kouvonen A, Vänskä J et al. Health, psychosocial factors and retirement intentions among Finnish physicians. Occup Med 2008;58(6):406-12.

9. Peterson U, Bergström G, Demerouti E et al. Burnout levels and self-rated health prospectively predict future long-term sickness absence: a study among female health professionals. J Occup Environ Med 2011;53(7):788-93.

10. Tountas $Y$, Manios $Y$, Dimitrakaki $C$ et al. Relationship between basic protective health behaviours and health related quality of life in Greek urban hospital employees. Int J Public Health 2007;52(6):341-7.

11. Abramson S, Stein J, Schaufele M et al. Personal exercise habits and counseling practices of primary care physicians: a national survey. Clin J Sport Med 2000;10(1):40-8.
12. Frank E, Segura C, Shen $\mathrm{H}$ et al. Predictors of Canadian physicians' prevention counseling practices. Can J Public Health 2010;101(5):390-5.

13. Lobelo F, Duperly J, Frank E. Physical activity habits of doctors and medical students influence their counseling practices. $\mathrm{Br} \mathrm{J}$ Sports Med 2009;43(2):89-92.

14. Lobelo F, De Quevedo IG. The evidence in support of physicians and health care providers as physical activity role models. Am J Lifestyle Med 2016;10(1):36-52.

15. Kelly M, Wills J, Sykes S. Do nurses' personal health behaviours impact on their health promotion practice? A systematic review. Int J Nurs Stud 2017;23(76):2376.

16. Blake H, Malik S, Mo PHK et al. 'Do as say, but not as I do': are next generation nurses role models for health? Perspect Public Health 2011;131(5):231-9.

17. Wills J, Kelly M. What works to encourage student nurses to adopt healthier lifestyles? Findings from an intervention study. Nurse Educ Today 2017;48:180-4.

18. World Health Organization. The international network of health promoting hospitals and health services: integrating health promotion into hospitals and health services (Concept, framework and organization). Copenhagen: World Health Organization; 2007.

19. Kosteva ARB, Salata BM, Krishnan SM et al. Physician variation in perceived barriers to personal health. Int J Gen Med 2012;5:53-7.

20. Mo PKH, Blake H, Batt ME. Getting healthcare staff more active: the mediating role of selfefficacy. Br J Health Psychol 2011;16(4):690706.

21. Borland R. CEOS theory: a comprehensive approach to understanding hard to maintain behaviour change. Appl Psychol Health Well Being 2017;9(1):3-35.

22. Schwarzer R. Modeling health behavior change: how to predict and modify the adoption and maintenance of health behaviors. J Appl Psychol 2008;57(1):1-29.
23. Hicks VL, Stolarczyk LM, Heyward VH et al. Validation of near-infrared interactance and skinfold methods for estimating body composition of American Indian women. Med Sci Sports Exerc 2000;32(2):531-9.

24. Aalto $M$, Tuunanen $M$, Sillanaukee $P$ et al. Effectiveness of structured questionnaires for screening heavy drinking in middle-aged women. Alcohol Clin Exp Res 2006;30(11):1884-8.

25. Tuunanen M, Aalto M, Seppä K. Binge drinking and its detection among middle-aged men using Audit, Audit-C and Audit-3. Drug Alcohol Rev 2007;26(3):295-9.

26. Murto J, Kaikkonen R, Pentala-Nikulainen O et al. National FinSote Survey. Available online at: https://www.thl.fi/en/web/thlfi-en/researchand-expertwork/population-studies/nationalfinsote-survey

27. Harmon-Jones E, Mills J. An introduction to cognitive dissonance and an overview of current perspectives on the theory. In: Harmon-Jones E, Mills J (eds). Cognitive dissonance: progress on a pivotal theory in social psychology. Washington, DC: American Psychological Association; 1999, pp. 3-21.

28. Ailasmaa R. Health care and social welfare personnel 2013. Statistical Report, National Institute for Health and Welfare, Helsinki, 2015. Available online at: http://urn.fi/ URN:NBN:fi-fe2015121023438

29. Wadden TA, Webb VL, Moran $\mathrm{CH}$ et al. Lifestyle modification for obesity: new developments in diet, physical activity, and behavior therapy. Circulation 2012;125:115770.

30. Shahar DR, Henkin Y, Rozen GS et al. A controlled intervention study of changing health-providers' attitudes toward persona lifestyle habits and health-promotion skills. Nutrition 2009;25(5):532-9.

31. Merrill RM, Anderson A, Thygerson SM. Effectiveness of a worksite wellness program on health behaviors and personal health. J Occup Environ Med 2011;53(9):1008-12. 